\title{
Ação anti-helmíntica de diferentes formulações de lactonas macrocíclicas em cepas resistentes de nematódeos de bovinos ${ }^{1}$
}

\author{
Alfredo Skrebsky Cezar ${ }^{*}$, Fernanda S.F. Vogel ${ }^{3}$, Luís Antônio Sangioni ${ }^{3}$, Ana \\ Maria Antonello ${ }^{4}$, Giovana Camillo ${ }^{2}$, Gustavo Toscan ${ }^{4}$ e Luciana O. de Araujo ${ }^{4}$
}

\begin{abstract}
Cezar A.S., Vogel F.S.F., Sangioni L.A., Antonello A.M., Camillo G., Toscan G. \& Araujo L.O. 2010. [Anthelmintic action of different formulations of macrocyclic lactones on resistant strains of nematodes of cattle.] Ação anti-helmíntica de diferentes formulações de lactonas macrocíclicas em cepas resistentes de nematódeos de bovinos. Pesquisa Veterinária Brasileira 30(7):523-528. Programa de Pós-Graduação em Medicina Veterinária Preventiva, Universidade Federal de Santa Maria (UFSM), Santa Maria, RS 97105-900, Brazil. E-mail: alfredosps@ hotmail.com

The macrocyclic lactones (MLs) (avermectins and milbemycins) are endectocides broadly used in livestock and in some parasitic diseases of humans. In cattle, parasite resistance to MLs is emerging, and the appearance of formulations that differ in their pharmacological properties become complex the choice of the most appropriate drug to each case. In order to evaluate possible alternatives to restore the effectiveness of MLs on resistant strains of gastrointestinal nematodes, were tested, in this study, ten different treatments based on the MLs on a population of gastrointestinal nematodes of cattle which, known, was under pressure of selection by $1 \%$ avermectins. Additionally, was tested a benzimidazole. The efficacy of the drugs was calculated with basis on the reduction of eggs per gram of feces (EPG) of cattle. The resistance of each genus was evaluated by identification of the larvae, obtained from culture in the feces, pre- and post-treatments. The desired efficacy was not obtained using long action avermectins - with high concentration and in association - even with the application of high doses. The genera Cooperia spp., Haemonchus spp. and Trichostrongylus spp. were resistant to avermectins, and Ostertagia spp. to ivermectin. It was observed that, once established parasite resistance to the $1 \%$ MLs, the application of drugs, of this same chemical group, even in formulations of high concentration, association or in high doses, may not result in the expected efficacy.
\end{abstract}

INDEX TERMS: Macrolactones, long action, parasite resistance, helminthes.

RESUMO.- As lactonas macrocíclicas (LMs) (avermectinas e milbemicinas) são endectocidas amplamente utilizados em animais e em algumas parasitoses humanas. Em bovinos, a resistência parasitária às LMs é emergente, e o surgimento de formulações que diferem nas suas propriedades

\footnotetext{
${ }^{1}$ Recebido em 10 de agosto de 2009.

Aceito para publicação em 11 de janeiro de 2010.

2 Programa de Pós-Graduação em Medicina Veterinária Preventiva, Doenças Parasitárias, Universidade Federal de Santa Maria (UFSM), Av. Roraima 1000, Prédio 44, Sala 5149, Camobi, Santa Maria, RS 97105-900, Brasil. "Autor para correspondência: alfredosps@ hotmail.com

${ }^{3}$ Departamento de Medicina Veterinária Preventiva (DMVP), Centro de Ciências Rurais (CCR), UFSM, Av. Roraima 1000, Prédio 44, Camobi, Santa Maria, RS 97105-900.

${ }^{4}$ Curso de Medicina Veterinária, UFSM, Santa Maria, RS 97105-900.
}

farmacológicas tornou complexa a escolha da droga mais indicada a cada caso. Com o objetivo de avaliar possíveis alternativas para recuperar a eficácia de LMs sobre cepas resistentes de nematódeos gastrintestinais, testaram-se, neste estudo, dez diferentes tratamentos a base de LMs sobre uma população de nematódeos gastrintestinais de bovinos a qual, sabidamente, sofrera pressão de seleção por avermectinas a $1 \%$. Adicionalmente, testou-se um benzimidazol. A eficácia das drogas foi calculada com base na redução de ovos por grama de fezes (OPG) dos bovinos. A resistência de cada gênero foi avaliada por meio de identificação de larvas, obtidas de cultivos nas fezes, pré- e pós-tratamentos. Não se obteve a eficácia desejada com o emprego de avermectinas 
de longa ação - com alta concentração e em associação - ou mesmo, com a aplicação de superdoses. Os gêneros Cooperia spp., Haemonchus spp. e Trichostrongylus spp. foram resistentes às avermectinas, e Ostertagia spp. à ivermectina. Observou-se que, uma vez estabelecida a resistência parasitária a LMs a 1\%, a aplicação de fármacos, deste mesmo grupo químico, ainda que em formulações mais concentradas, asso-ciações ou superdoses, pode não resultar na eficácia esperada.

TERMOS DE INDEXAÇÃO: Macrolactonas, longa ação, resistência parasitária, helmintos.

\section{INTRODUÇÃO}

As infecções por nematódeos do trato gastrintestinal influenciam negativamente na produtividade e no bem-estar dos bovinos, sendo necessário que se promovam medidas de controle e profilaxia que as minimizem. Até o momento, quase todos os métodos de controle dessas parasitoses dependem, em algum grau, do emprego de quimioterápicos. As lactonas macrocíclicas (LMs) são endectocidas de amplo espectro (avermectinas e milbemicinas), largamente utilizados em animais domésticos e em algumas parasitoses de humanos (Shoop et al. 1995, Geary 2005). A resistência parasitária a essas drogas encontra-se disseminada ao redor do mundo nos rebanhos bovinos (Kaplan 2004), reduzindo a eficácia e, por conseqüência, o retorno econômico dos tratamentos antiparasitários.

As LMs disponíveis para aplicação em bovinos apresentam-se em diversas formulações - com diferentes princípios ativos, concentrações e associações - dando alternativas ao produtor para a escolha de drogas eficazes no seu rebanho e adequadas ao seu manejo. Porém, é necessário que se encontrem meios para estender a vida-útil destes e dos demais fármacos antiparasitários, caso contrário, o desenvolvimento de resistência múltipla aos anti-helmínticos pode dificultar, ou mesmo, inviabilizar o controle das nematodíases no rebanho (Coles 2002, Kaplan 2004). Além disso, deve-se destacar a importância de manter-se a viabilidade dos endectocidas para programas de controle integrado das parasitoses.

Há correlação positiva entre a dose administrada e a concentração plasmática de LMs em bovinos (Lanusse \& Prichard 1993, van Zeveren et al. 2007) e alguns estudos indicam que a aplicação de LMs de alta concentração com longa ação (LA) e em associação pode aumentar a eficiência dos tratamentos (Dobson et al. 1996, Catto et al. 2005, Yazwinski et al. 2006, Borges et al. 2008). Além disso, na seleção experimental de nematódeos resistentes a LMs, a partir de cepas originalmente susceptíveis, em geral, é necessária a aplicação de uma subdose para desencadear o processo de seleção, a qual é aumentada gradativamente, sobre subseqüentes gerações do parasita, até obterem-se cepas resistentes à dose terapêutica do princípio ativo (Ranjan et al. 2002, van Zeveren et al. 2007). Essas observações apontam para uma adaptação dose-dependente da população parasitária ao princípio ativo, o que indica que cepas resistentes à dose terapêutica mínima de determinada droga poderiam ser susceptíveis ao contato com maiores con- centrações da mesma. Levando-se em conta esses fatos, e as evidências de que as cepas resistentes presentes nos rebanhos podem diferir daquelas produzidas em laboratório (Gill et al. 1998), torna-se importante a investigação criteriosa da aplicabilidade desses conceitos em condições de campo.

Nesse sentido, o objetivo deste estudo foi avaliar a ação de LMs - com diferentes princípios ativos e concentrações, em associação, com diferentes doses e períodos de persistência - e, adicionalmente, de um fármaco do grupo dos benzimidazóis, sobre uma população de helmintos que, sabidamente, sofrera pressão de seleção por avermectinas a $1 \%$, e a qual não sofrera pressão de seleção por outros anti-helmínticos, nos 8 anos anteriores ao estudo. Com isso, buscou-se avaliar a viabilidade dessas alternativas para o controle de helmintos em uma população resistente às avermectinas de baixa concentração (a 1\%).

\section{MATERIAL E MÉTODOS}

Utilizou-se um rebanho bovino situado no município de São Pedro do Sul, no estado do Rio Grande do Sul, Brasil, cuja escolha deveu-se ao manejo antiparasitário previamente realizado, que consistia na aplicação de doramectina a 1\% (DRM $1 \%$ ) (apenas em neonatos ou pré-castração - nos machos) e de ivermectina a 1\% (IVM 1\%), com média de 4 tratamentos/ ano, em todas as categorias animais. Não havia critério definido para os tratamentos, sendo, os mesmos, realizados de maneira profilática e, por conveniência, quando os animais viessem a ser manejados para outros fins. Assim, por conta da pressão de seleção exercida sobre os nematódeos gastrintestinais presentes nos bovinos e, por conseqüência, nas pastagens, partiu-se de boa probabilidade de se encontrarem cepas de nematódeos resistentes à LMs. Deste rebanho, utilizou-se um lote de 149 bovinos da raça Brangus, com cerca de 12 meses de idade, mantidos em campo nativo, com densidade de 1,1 UA ha-1 e isentos de tratamento antihelmíntico nos três meses precedentes ao início dos testes.

$\mathrm{O}$ estudo constituiu-se de três experimentos subseqüentes e interdependentes, onde os resultados de cada experimento indicavam o que seria testado a seguir. Durante o curso deste estudo avaliou-se, pelos mesmos métodos, um grupo de animais não-tratados junto a cada um dos experimentos. Com isso, monitoraram-se possíveis variações naturais da população parasitária que pudessem interferir nos resultados dos testes de eficácia.

Experimento 1: testou-se a eficácia anti-helmíntica de avermectinas e de uma milbemicina, nas suas formulações a $1 \%$, em veículo oleoso (de liberação lenta) ou não-aquoso. Adicionalmente, testou-se a eficácia de um anti-helmíntico do grupo dos benzimidazóis. Para isso, constituíram-se 6 grupos $(n=8)$, cujos tratamentos foram: G1. ivermectina a $1 \% 5$ (IVM $1 \%)(0,2 \mathrm{mg}$ $\left.\mathrm{kg}^{-1}\right)$; G2. doramectina a $1 \% 6$ (DRM 1\%) $\left(0,2 \mathrm{mg} \mathrm{kg}^{-1}\right)$; G3. ivermectina a $1 \% \mathrm{LA}^{7}$ (IVM 1\% LA) $\left(0,2 \mathrm{mg} \mathrm{kg}^{-1}\right)$; G4. abamectina a $1 \% \mathrm{LA}^{8}$ (ABM $1 \%$ LA) $\left(0,2 \mathrm{mg} \mathrm{kg}^{-1}\right)$; G5. moxidectina a $1 \%{ }^{9}$ (MOX 1\%) $\left(0,2 \mathrm{mg} \mathrm{kg}^{-1}\right)$; G6. sulfóxido de albendazol a $10 \%{ }^{10}$ (SULF-ALB 10\%) (2,5 $\mathrm{mg} \mathrm{kg}^{-1}$ ) - do grupo dos benzimidazóis.

Experimento 2: testou-se a ação anti-helmíntica de avermectinas de alta concentração com longa ação (LA), incluindo-se uma droga composta por uma associação de princípios ativos, sobre a população de nematódeos gastrintestinais resistente às avermectinas a $1 \%$. Os grupos tratados $(n=8)$ 
foram: G7. ivermectina a 3,15\% LA ${ }^{11}$ (IVM 3,15\% LA) $(0,63 \mathrm{mg}$ $\left.\mathrm{kg}^{-1}\right)$; G8. ivermectina a $4 \% \mathrm{LA}^{12}$ (IVM $4 \%$ LA) $\left(0,8 \mathrm{mg} \mathrm{kg}^{-1}\right)$; G9. ivermectina a $2,25 \%\left(0,45 \mathrm{mg} \mathrm{kg}^{-1}\right)+$ abamectina a $1,25 \% \mathrm{LA}^{13}$ $\left(0,25 \mathrm{mg} \mathrm{kg}^{-1}\right)(\mathrm{IVM}+\mathrm{ABM} 3,5 \% \mathrm{LA})$.

Experimento 3: testou-se a ivermectina - a $1 \%$ e a 3,15\% LA em superdoses (triplo da dose terapêutica) sobre a população de nematódeos gastrintestinais resistente às avermectinas a $1 \%$. Os grupos tratados $(\mathrm{n}=8)$ foram: G10. IVM $1 \% 5$ em dose tripla $(0,6 \mathrm{mg}$ $\left.\mathrm{kg}^{-1}\right)$; G11. IVM 3,15\% LA ${ }^{11}$ em dose tripla $\left(1,9 \mathrm{mg} \mathrm{kg}^{-1}\right)$.

Procederam-se coletas de fezes no dia anterior ao tratamento (D-1) e aos $7(D+7), 14(D+14)$ e $21(D+21)$ dias póstratamento. Realizaram-se contagens de ovos de nematódeos por grama de fezes (OPG) (individualmente para cada amostra fecal), pelo método de Gordon \& Withlock, modificado, com sensibilidade de detecção de 50 OPG; e cultivos de larvas (uma por grupo experimental, em cada uma das coletas), pelo método de Roberts \& O'Sullivan, identificando-se até 100 larvas infectantes por coprocultura (Ueno \& Gonçalves 1998). Foram selecionados animais com OPG inicial $\geq 200$ (D-1), os quais foram divididos em grupos com médias de OPG pré-tratamento equivalentes, a cada experimento, por meio de randomização estratificada. Todas as drogas testadas foram administradas pela via subcutânea, em dose única. Os dias dos tratamentos foram tomados como dias zero (D0) dos experimentos.

Seguindo as recomendações da World Association for the Advancement of Veterinary Parasitology (Coles et al. 1992), considerou-se eficaz aquela droga que atingiu ou superou os 95\% de redução de OPG aos $7(D+7)$ e aos $14(D+14)$ dias pós-tratamento, calculado pela fórmula: $P R=100$ (1 - OPGpós / OPGpré), onde, "PR" representa o percentual de redução de OPG calculado; "OPGpós" equivale à média aritmética de OPG para cada grupo pós-tratamento $(D+7, D+14$ e $D+21)$; e "OPGpré" equivale à média aritmética de OPG para cada grupo pré-tratamento (D-1). Os valores de OPG foram comparados pelo teste ANOVA com DMS Duncan, com nível de significância de 5\%. O grau de resistência, de cada gênero de nematódeo presente no rebanho para cada droga testada, foi avaliado pela comparação entre o seu percentual representativo, nos cultivos de larvas, pré- e pós-tratamento.

\section{RESULTADOS E DISCUSSÃO}

No Quadro 1 são demonstradas as médias de OPG e as eficácias calculadas das formulações testadas. Devido a nenhum

\footnotetext{
${ }^{5}$ Ivermectina $1 \%$ Ouro Fino, Ouro Fino Saúde Animal, Rodovia Anhanguera SP $330 \mathrm{Km}$ 298, Cravinhos, SP.

${ }^{6}$ Dectomax®, Pfizer Saúde Animal, Avenida Monteiro Lobato 2270, Guarulhos, SP.

${ }^{7}$ Ivotan® L.A., Intervet do Brasil Veterinária Ltda, Avenida Sir Henry Wellcome 335, Cotia, SP.

${ }^{8}$ Abamectina 1\% Ouro Fino LA, Ouro Fino Saúde Animal, Rodovia Anhanguera SP $330 \mathrm{Km}$ 298, Cravinhos, SP.

${ }^{9}$ Cydectin Injetável $($, Fort Dodge Saúde Animal, Rua Luiz Fernando Rodriguez, 1701, Campinas, SP.

${ }^{10}$ Ricobendazole 10 Injetável, Ouro Fino Saúde Animal, Rodovia Anhanguera SP $330 \mathrm{Km}$ 298, Cravinhos, SP.

${ }^{11}$ Ivomec@ Gold, Merial Saúde Animal, Fazenda São Francisco s/n, Paulínia, SP.

${ }^{12}$ Master LP, Ouro Fino Saúde Animal, Rodovia Anhanguera SP 330 Km 298, Cravinhos, SP.

${ }^{13}$ Solution ${ }^{\circledR} 3,5 \%$ L.A., Intervet do Brasil Veterinária LTDA, Avenida Sir Henry Wellcome 335, Cotia, SP.
}

dos tratamentos testados durante este estudo ter sido capaz de manter uma média de redução de $\mathrm{OPG} \geq 95 \%$ (em relação à D-1) até o D+21 (dados não demonstrados), não foram realizadas novas coletas depois de decorrido este período.

Conforme se observa no Quadro 1, detectou-se resistência parasitária aos fármacos que já vinham sendo utilizados nos bovinos - IVM 1\% (G1) e DRM 1\% (G2) - e resistência lateral à $A B M(G 4)$ (princípio ativo que não houvera sido utilizado anteriormente neste rebanho). No que diz respeito às médias de OPG pós-tratamentos, tanto a DRM quanto a ABM apresentaram melhor desempenho no $D+7(p<0,05)$, se comparado às IVMs a 1\% (G1 e G3). A resistência parasitária à IVM $1 \%$ já era esperada neste caso, uma vez que esta era a formulação mais freqüentemente utilizada nos bovinos. 0 desenvolvimento de resistência às avermectinas, em rebanhos tratados profilaticamente com esses fármacos (Soutello et al. 2007, Souza et al. 2008), e a ocorrência de resistência lateral entre princípios ativos desse grupo químico (Mello et al. 2006) têm sido comumente descritos. Por outro lado, não se observou, neste estudo, resistência lateral à MOX $1 \%$ (G5), diferentemente do que fora relatado por Vermunt et al. (1996). Embora avermectinas e milbemicinas apresentem mecanismos de ação quase idênticos (Shoop et al. 1995), o que, aparentemente, implica em que o desenvolvimento de resistência às avermectinas provoque, paralelamente, resistência às milbemicinas e vice-versa (Kaplan et al. 2007), pouco se sabe a respeito de quais fatores poderiam ser determinantes para que ocorra ou não resistência lateral para avermectinas/milbemicinas em uma população parasitária. Segundo Ranjan et al. (2002) a resistência parasitária à MOX pode se desenvolver mais lentamente, em comparação com a IVM, na população parasitária, fazendo com que parasitas resistentes à IVM permaneçam susceptíveis à dose terapêutica da MOX. Essas observações vão ao encontro dos resultados deste estudo, cuja população parasitária fora selecionada pela dose terapêutica de avermectinas. Todavia, Condi et al. (2009) demonstraram que a resistência parasitária à MOX, avaliada pela redução da contagem de OPG, pode acabar sendo subestimada devido à supressão, provocada pela droga, na excreção de ovos pelos nematódeos gastrintestinais, sem que haja, porém, a equivalente redução da carga parasitária. Por conta disso, deve-se ter cautela ao utilizar-se a MOX em caso de ineficácia de outras LMs em um rebanho, uma vez que a diferença observada na eficácia da MOX em relação às avermectinas será, possivelmente, temporária, sendo que o modelo experimental utilizado não permitiu a comprovação desta tendência pelo curto período de observação.

Conforme se demonstra no Quadro 1, embora tenham sido observados maiores percentuais de redução de $O P G$ com a aplicação de IVM 3,15\% LA (G7) e de IVM 4\% LA (G8), se comparado às IVMs a 1\% (G1 e G3), não houve diferença estatística para as médias de OPG pós-tratamentos entre estes grupos, exceto no caso do G3, no qual o desempenho do tratamento foi inferior ao do $\mathrm{G} 7$ e ao do G8 $(p<0,05)$ no $D+14$. Por outro lado, a associação IVM+ABM 3,5\% LA (G9) determinou menores médias de 


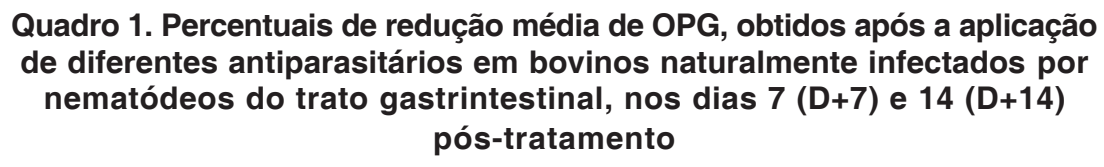

\begin{tabular}{|c|c|c|c|}
\hline \multirow[t]{2}{*}{ Grupos e tratamentos (doses) } & \multicolumn{3}{|c|}{ Médias de OPG (PR) } \\
\hline & D-1 & $\mathrm{D}+7$ & $\mathrm{D}+14$ \\
\hline G1. I & $494^{\mathrm{A}}$ a & $\mathrm{a} b \mathrm{~b}(1$ & $356^{\mathrm{A}}$ a b $(28 \%)$ \\
\hline $\mathrm{G}$ & $506^{\mathrm{A} a}$ & $125^{\mathrm{B} d}(75 \%)$ & $181^{\mathrm{B}}$ b c $(64 \%)$ \\
\hline G3. I & $531^{\mathrm{A}}$ a & $456^{\mathrm{A}}$ a $(14 \%)$ & $544^{A}$ a $(-2 \%)$ \\
\hline G4. $A$ & $506^{\mathrm{A} \text { a }}$ & $206^{A}$ c d $(59 \%)$ & $175^{\mathrm{A}}$ b c $(65 \%)$ \\
\hline G5 & $494^{\mathrm{A}}$ a & $0^{B ~ d}(100 \%)^{*}$ & $025^{\mathrm{B}} \mathrm{c}(95 \%)^{*}$ \\
\hline$\%\left(2,5 \mathrm{mg} \mathrm{kg}^{-1}\right)$ & $544^{\mathrm{A}}$ a & $019^{\mathrm{B} d}(97 \%)^{*}$ & $019^{\mathrm{B}} \mathrm{c}(97 \%)^{*}$ \\
\hline$\left(0,63 \mathrm{mg} \mathrm{kg}^{-1}\right)$ & $512^{\mathrm{A} a}$ & $412^{\mathrm{A}}$ a b ${ }^{\mathrm{C}}(20 \%)$ & $175^{\mathrm{B}}$ b c $(66 \%)$ \\
\hline G8. IVM 4 & $475^{\mathrm{A}}$ a & $219^{\mathrm{B}}$ b c d $(54 \%)$ & $175^{\mathrm{B}}$ b c $(63 \%)$ \\
\hline G9. IVM+ABM 3,5 & $506^{\mathrm{A} a}$ & $144^{\mathrm{A} d}(71 \%)$ & $156^{\mathrm{A}} \mathrm{b} c \mathrm{(69 \% )}$ \\
\hline & $487^{\mathrm{A} \text { a }}$ & $161^{\mathrm{B} d}(67 \%)$ & $487^{\mathrm{A}}$ a $(0 \%)$ \\
\hline G11. IVM 3 & $506^{\mathrm{A}}$ a & $219^{A}$ b c d $(57 \%)$ & $225^{\mathrm{A}}$ b c $(56 \%)$ \\
\hline
\end{tabular}

$\overline{\mathrm{PR}}=$ percentual de redução de OPG por grupo em $\mathrm{D}+7$ e $\mathrm{D}+14$ em relação à $\mathrm{D}-1$.

* Tratamentos eficazes: PR $\geq 95 \%$ (Coles et al. 1992).

Letras diferentes, na mesma linha (maiúsculas) ou na mesma coluna (minúsculas), indicam diferença estatística, pelo teste ANOVA com DMS Duncan, com $p<0,05$.

OPG $(p<0,05)$ tanto em relação à IVM a $1 \%(D+7)$ como à IVM $1 \%$ LA ( $D+7$ e $D+14)$. Além disso, o desempenho dessa associação (G9) foi superior $(p<0,05)$ ao da IVM $3,15 \%$ LA (G7) no D+7. Contudo, estes tratamentos com formulações de alta concentração não atingiram a eficácia desejada (redução média de OPG $\geq 95 \%$ ) (Coles et al. 1992), o que não foi obtido mesmo com a aplicação de superdosagens de IVM (G10 e G11), apesar da obtenção de menores médias de OPG $(p<0,05)$ no $D+7$ para o $G 10$ em relação ao $\mathrm{G} 1$ e ao $\mathrm{G} 3$, e no $\mathrm{D}+14$ para o $\mathrm{G} 11$ se comparado ao G3.

De forma geral, pode-se afirmar que os nematódeos gastrintestinais dos bovinos, os quais já se mostravam resistentes aos tratamentos realizados na propriedade, apresentaram resistência a uma concentração equivalente ao triplo da dose terapêutica recomendada para a IVM.

O SULF-ALB10\% foi eficaz, considerando-se a redução média de OPG (D+7 e $D+14)$. Em uma primeira análise, estes resultados não confirmariam as evidências de que a pressão de seleção, exercida pelas LMs, seria ca- paz de aumentar a freqüência de alelos envolvidos na resistência aos benzimidazóis na população parasitária, como comprovado, in vitro, na espécie Haemonchus contortus (Mottier \& Prichard 2008). Porém, recuperaram-se larvas resistentes a este tratamento (Quadro 2), com alta predominância de Haemonchus spp. no D+7, seguida por grande representatividade de Cooperia spp. no D+14.

Não houve variações representativas das médias de OPG dos grupos não-tratados no curso dos experimentos. Nestes grupos os gêneros Cooperia spp. e Haemonchus spp. predominaram, as freqüências de Trichostrongylus spp. e Ostertagia spp. foram consideráveis e de Oesophagostomum spp. foram mínimas (dados não-demonstrados).

Conforme observa-se no Quadro 2, o gênero Oesophagostomum spp. não se demonstrou resistente a nenhum dos tratamentos. Após o tratamento (D+14) com MOX 1\% (G5), sobressaíram-se larvas de Haemonchus spp. e Cooperia spp. (Quadro 2), porém, a elevada redução de OPG obtida com esta droga (Quadro 1) não permitiu a com-

Quadro 2. Representatividade de larvas infectantes (L3) recuperadas dos cultivos de larvas - no dia anterior aos tratamentos (-1) e nos dias $7(+7)$ e $14(+14)$ pós-tratamentos - para os gêneros Cooperia spp. (Coop.), Haemonchus spp. (Haem.), Ostertagia spp. (Ostert.), Trichostrongylus spp. (Trich.) e Oesophagostomum spp. (Oesoph.)

\begin{tabular}{|c|c|c|c|c|c|c|c|c|c|c|c|c|c|c|c|}
\hline \multirow[t]{2}{*}{ Grupos e tratamentos (doses) } & \multicolumn{3}{|c|}{ Coop. (\%) } & \multicolumn{3}{|c|}{ Haem. (\%) } & \multicolumn{3}{|c|}{ Ostert. (\%) } & \multicolumn{3}{|c|}{ Trich. (\%) } & \multicolumn{3}{|c|}{ Oesoph. (\%) } \\
\hline & -1 & +7 & -14 & -1 & +7 & +14 & -1 & +7 & +14 & & +7 & +14 & -1 & +7 & +14 \\
\hline G1. IVM $1 \%\left(0,2 \mathrm{mg} \mathrm{kg}^{-1}\right)$ & 37 & 63 & 61 & 40 & 35 & 38 & 3 & 0 & 0 & 19 & 2 & 1 & 1 & 0 & 0 \\
\hline G2. DRM $1 \%\left(0,2 \mathrm{mg} \mathrm{kg}^{-1}\right)$ & 28 & 65 & 79 & 58 & 34 & 20 & 3 & 1 & 0 & 9 & 0 & 1 & 2 & 0 & 0 \\
\hline G3. IVM $1 \%$ LA $\left(0,2 \mathrm{mg} \mathrm{kg}^{-1}\right)$ & 68 & 91 & 90 & 14 & 6 & 7 & 4 & 0 & 0 & 14 & 3 & 3 & 0 & 0 & 0 \\
\hline G4. ABM $1 \%$ LA $\left(0,2 \mathrm{mg} \mathrm{kg}^{-1}\right)$ & 52 & 48 & 74 & 27 & 52 & 23 & 0 & 0 & 0 & 20 & 0 & 3 & 1 & 0 & 0 \\
\hline G5. MOX $1 \%\left(0,2 \mathrm{mg} \mathrm{kg}^{-1}\right)$ & 78 & 0 & 56 & 16 & 0 & 40 & 0 & 0 & 0 & 6 & 0 & 4 & 0 & 0 & 0 \\
\hline G6. SULF-ALB $10 \%\left(2,5 \mathrm{mg} \mathrm{kg}^{-1}\right)$ & 81 & 10 & 69 & 8 & 90 & 31 & 1 & 0 & 0 & 9 & 0 & 0 & 1 & 0 & 0 \\
\hline G7. IVM $3,15 \%$ LA $\left(0,63 \mathrm{mg} \mathrm{kg}^{-1}\right)$ & 72 & 70 & 57 & 23 & 28 & 43 & 0 & 0 & 0 & 5 & 2 & 0 & 0 & 0 & 0 \\
\hline G8. IVM $4 \%$ LA $\left(0,8 \mathrm{mg} \mathrm{kg}^{-1}\right)$ & 67 & 14 & 28 & 28 & 86 & 72 & 0 & 0 & 0 & 5 & 0 & 0 & 0 & 0 & 0 \\
\hline G9. IVM+ABM $3,5 \%$ LA $\left(0,7 \mathrm{mg} \mathrm{kg}^{-1}\right)$ & 42 & 14 & 28 & 51 & 86 & 72 & 0 & 0 & 0 & 7 & 0 & 0 & 0 & 0 & 0 \\
\hline G10. IVM $1 \%\left(0,6 \mathrm{mg} \mathrm{kg}^{-1}\right)$ & 8 & 16 & 18 & 71 & 76 & 68 & 10 & 6 & 8 & 2 & 2 & 6 & 9 & 0 & 0 \\
\hline G11. IVM 3,15\% LA $\left(1,9 \mathrm{mg} \mathrm{kg}^{-1}\right)$ & 4 & 4 & 48 & 78 & 90 & 38 & 7 & 4 & 0 & 4 & 2 & 14 & 7 & 0 & 0 \\
\hline
\end{tabular}


provação de resistência da população parasitária neste caso. Por outro lado, conforme se observa no Quadro 2, os gêneros Cooperia spp. e Haemonchus spp. demonstraram resistência a todos os tratamentos com avermectinas, larvas do gênero Trichostrongylus spp. resistentes às avermectinas também foram recuperadas ao longo deste estudo, e o gênero Ostertagia spp., que apareceu em níveis consideráveis apenas no Experimento 3, demonstrou resistência à IVM (G10 e G11). Nota-se que, tal como relatado neste, cepas de Cooperia spp. e Haemonchus spp. resistentes às avermectinas têm sido comumente descritas em bovinos (Rangel et al. 2005, Soutello et al. 2007, Suarez \& Cristel 2007, Borges et al. 2008). Em relação ao gênero Cooperia spp., isto pode estar relacionado à sua característica de ser dose-limitante para as LMs (Shoop et al. 1995), ou seja, de ser naturalmente mais tolerante a esses fármacos se comparado a maioria dos demais gêneros de nematódeos. Para o gênero Haemonchus spp., tal fato parece estar relacionado à dominância genética que torna mais eficiente a transmissão da resistência às LMs (Dobson et al. 1996). Já no que confere aos gêneros Trichostrongylus spp. e Ostertagia spp., os relatos de resistência às LMs em rebanhos bovinos são mais raros, e os mecanismos envolvidos neste processo são pouco conhecidos.

A concentração necessária nos tecidos-alvos, para que a droga seja eficaz, é variável entre os gêneros de helmintos (Lifschitz et al. 1999), o que sugere que possa variar também entre cepas resistentes e susceptíveis de um mesmo gênero de nematódeo (Shoop et al. 1995). As farmacocinéticas da IVM $1 \%$ e da IVM 3,15\% LA foram demonstradas por Lifschitz et al. (2007), os quais observaram influências da concentração do fármaco, da dose aplicada e do veículo (não-aquoso ou oleoso) na curva de concentração plasmática da IVM em bovinos. Com base nessas informações, podese afirmar que ambas as formulações, nas doses usuais $\left(0,2 \mathrm{mg} \mathrm{kg}^{-1}\right.$ e $0,63 \mathrm{mg} \mathrm{kg}^{-1}$, respectivamente), apresentam picos plasmáticos equivalentes, devido à liberação lenta da IVM em veículo oleoso (caso da IVM 3,15\% LA). No presente estudo, as formulações de IVM de alta concentração com liberação lenta (G7 e G8), em geral, determinaram maior redução média de OPG sobre as cepas resistentes em relação às IVMs a 1\% (G1 e G3). Isto poderia indicar que o tempo de exposição dos parasitas a uma mesma concentração plasmática do princípio ativo tem influência positiva na eficácia do tratamento.

Neste contexto, as formulações LA têm como principal vantagem sua maior persistência em concentração suficiente à manutenção da sua ação antiparasitária (Dobson et al. 1996). A exemplo disso, Catto et al. (2005) detectaram menor prevalência das infecções, menor média de OPG e maior ganho de peso em bezerros tratados com IVM 3,15\% LA em comparação a bezerros tratados com IVM 1\%, devendo-se ressaltar que essas diferenças puderam ser constatadas em um período observacional mais longo (dos 3 a 5 meses ao desmame). Porém, no presente estudo, a ação dessa maior persistência das formulações LA no controle da re-infecção parasitária não pôde ser quantificada, o que se deveu à baixa eficácia destes fármacos no rebanho utilizado. Adicionalmente, ao utilizar-se uma associação de avermectinas, como no caso da IVM+ABM 3,5\% LA (G9), soma-se à maior persistência, um possível efeito combinado dos princípios ativos, levando-se em conta que há diferenças na susceptibilidade dos diferentes gêneros ou espécies de helmintos a cada uma dessas bases químicas (Borges et al. 2008). De outra forma, ao aumentar-se a dose administrada de IVM, tal qual realizado nos grupos G10 e G11, obtém-se um aumento equivalente do seu pico de concentração plasmática (Lifischitz et al. 2007), o que poderia ser determinante para a eficácia do princípio ativo sobre helmintos menos sensíveis (ou mais resistentes) ao mesmo.

Juntamente a essas observações, deve-se considerar o fato de que na seleção experimental, em condições laboratoriais, de genótipos de parasitas resistentes a LMs como, por exemplo, de H. contortus (Ranjan et al. 2002) e de Ostertagia ostertagi (van Zeveren et al. 2007), o processo de seleção é desencadeado pela aplicação de subdoses nos animais inoculados com a cepa parental (susceptível), seguido de aumento gradativo da dose sobre subseqüentes gerações dos parasitas, até a obtenção de uma cepa resistente à dose terapêutica do princípio ativo, devendo-se ressaltar que, nestes casos, não há o efeito da refugia e de outros fatores epidemiológicos que poderiam interferir na proporção de genótipos resistentes.

A conjunção destas observações permitia supor que, em condições de campo, ao sofrer pressão de seleção pela dose terapêutica (i.e. $0,2 \mathrm{mg} \mathrm{kg}^{-1}$ de IVM), durante um longo período, a população parasitária que se tornasse resistente, pudesse apresentar susceptibilidade a maiores doses e concentrações de princípio ativo, o que não se confirmou em termos de eficácia baseada na redução média de OPG, no presente estudo.

A baixa eficácia dos tratamentos anti-helmínticos, detectada neste rebanho, indica a ocorrência de perdas econômicas, as quais não estavam sendo identificadas pelos proprietários por decorrerem de infecções subclínicas. Esta situação, provavelmente, é similar à de outras propriedades que utilizam as LMs de maneira profilática, ou ainda, supressiva.

Buscar meios de restabelecer a eficácia das LMs em parasitas resistentes de animais pode ser uma alternativa para estender a vida útil deste importante grupo de endectocidas nos rebanhos e poderia, inclusive, servir de base para casos de resistência em parasitas de humanos, onde a recuperação clínica do paciente suplantaria maiores custos com os tratamentos. Porém, observou-se que, uma vez estabelecida a resistência parasitária às avermectinas a $1 \%$, a utilização destes fármacos, ainda que em formulações mais concentradas, associações ou superdoses, pode não resultar no retorno esperado, devendo-se testar a viabilidade destas opções no rebanhoalvo antes de instituírem-se tais tratamentos.

Restam, ainda, diversos pontos a serem esclarecidos em relação aos mecanismos de resistência parasitária, especialmente em condições de campo. É neces- 
sário que se aprofundem pesquisas nesta área visando um melhor aproveitamento das drogas antiparasitárias existentes no mercado.

\section{REFERÊNCIAS}

Borges F.A., Silva H.C., Buzzulini C., Soares V.E., Santos E., Oliveira G.P. \& Costa A.J. 2008. Endectocide activity of a new long-action formulation containing $2.25 \%$ ivermectin $+1.25 \%$ abamectin in cattle. Vet. Parasitol. 155(3/4):299-307.

Catto J.B., Bianchin I. \& Torres Junior R.A.A. 2005. Efeitos da everminação de matrizes e de bezerros lactentes em sistema de produção de bovinos de corte na região de Cerrado. Pesq. Vet. Bras. 25(3):188194.

Coles G.C. 2002. Cattle nematodes resistant to anthelmintics: Why so few cases? Vet. Res. 33(5):481-489.

Coles G.C., Bauer C., Borgsteede F.H.M., Geerts S., Klei T.R., Taylor M.A. \& Waller P.J. 1992. World Association for the Advancement of Veterinary Parasitology (W.A.A.V.P.) methods for the detection of anthelmintic resistance in nematodes of veterinary importance. Vet. Parasitol. 44(1/2):35-44.

Condi G.K., Soutello R.G.V. \& Amarante A.F.T. 2009. Moxidectin-resistant nematodes in cattle in Brazil. Vet. Parasitol. 161(3/4):213-217.

Dobson R.J., Lejambre L. \& Gill J. 1996. Management of anthelmintic resistance: Inheritance of resistance and selection with persistent drugs. Int. J. Parasitol. 26(8/9):993-1000.

Geary T.G. 2005. Ivermectin 20 years on: Maturation of a wonder drug. Trends Parasitol. 21(11): 530-532.

Gill J.H., Kerr C.A., Shoop W.L. \& Lacey E. 1998. Evidence of multiple mechanisms of avermectin resistance in Haemonchus contortus: Comparison of selection protocols. Int. J. Parasitol. 28(5):783-789.

Kaplan R.M. 2004. Drug resistance in nematodes of veterinary importance: A status report. Trends Parasitol. 20(10):477-481.

Kaplan R.M., Vidyashankar A.N., Howell S.B., Neiss J.M., Williamson L.H. \& Terrill T.H. 2007. A novel approach for combining the use of in vitro and in vivo data to measure and detect emerging moxidectin resistance in gastrointestinal nematodes of goats. Int. J. Parasitol. 37(7):795-804

Lanusse C.E. \& Prichard R.K. 1993. Relationship between pharmacological properties and clinical efficacy of ruminant anthelmintics. Vet. Parasitol. 49(2/4):123-158.

Lifschitz A., Virkel G., Pis A., Imperiale F., Sanchez S., Alvarez L., Kujanek R. \& Lanusse C. 1999. Ivermectin disposition kinetics after subcutaneous and intramuscular administration of an oil-based formulation to cattle. Vet. Parasitol. 86(3):203-215.

Lifschitz A., Virkel G., Ballent M., Sallovitz J., Imperiale F., Pis A. \& Lanusse C. 2007. Ivermectin (3.15\%) long-action formulations in cattle: Absorption pattern and pharmacokinetic considerations. Vet. Parasitol. 147(3/4):303-310.

Mello M.H.A., Depner R., Molento M.B. \& Ferreira J.J. 2006. Resistência lateral às macrolactonas em nematodas de bovinos. Arch. Vet. Sci. 11(1):8-12.

Mottier M.L. \& Prichard R.K. 2008. Genetic analysis of a relationship between macrocyclic lactone and benzimidazole anthelmintic selection on Haemonchus contortus. Pharmacogenet. Genomics 18(2):129-140.

Rangel V.B., Leite R.C., Oliveira P.R. \& Santos Junior E.J. 2005. Resistência de Cooperia spp. e Haemonchus spp. às avermectinas em bovinos de corte. Arq. Bras. Med. Vet. Zoot. 57(2):186-190.

Ranjan S., Wang G.T., Hirschlein C. \& Simkins K.L. 2002. Selection for resistance to macrocyclic lactones by Haemonchus contortus in sheep. Vet. Parasitol. 103(1/2):109-117.

Shoop W.L., Mrozik H. \& Fisher M.H. 1995. Structure and activity of avermectins and milbemycins in animal health. Vet. Parasitol. 59(2):139-156.

Soutello R.G., Seno M.C.Z. \& Amarante A.F.T. 2007. Anthelmintic resistance in cattle nematodes in northwestern São Paulo State, Brazil. Vet. Parasitol. 148(3/4):360-364

Souza A.P., Ramos C.I., Bellato V., Sartor A.A. \& Schelbauer C.A. 2008. Resistência de helmintos gastrintestinais de bovinos a antihelmínticos no Planalto Catarinense. Ciên. Rur. 38(5):1363-1367.

Suarez V.H. \& Cristel S.L. 2007. Anthelmintic resistance in cattle nematode in the western Pampeana Region of Argentina. Vet. Parasitol. 144(1/2):111-117.

Ueno H. \& Gonçalves V.C. 1998. Manual para diagnóstico das helmintoses de ruminantes. Japan International Cooperation Agency, Tóquio. 143p.

van Zeveren A.M., Casaert S., Alvinerie M., Geldhof P., Claerebout E. \& Vercruysse J. 2007. Experimental selection for ivermectin resistance in Ostertagia ostertagi in cattle. Vet. Parasitol. 150(1/2):104-110.

Vermunt J., West D. \& Pomroy W. 1996. Inefficacy of moxidectin and doramectin against ivermectin-resistant Cooperia spp. of cattle in New Zealand. N. Z. Vet. J. 44(5):188-193.

Yazwinski T.A., Williams J.C., Smith L.L., Tucker C., Loyacano A.F., DeRosa A., Peterson P., Bruer D.J. \& Delay R.L. 2006. Dose determination of the persistent activity of moxidectin long-acting injectable formulations against various nematode species in cattle. Vet. Parasitol. 137(3/4):273-285. 